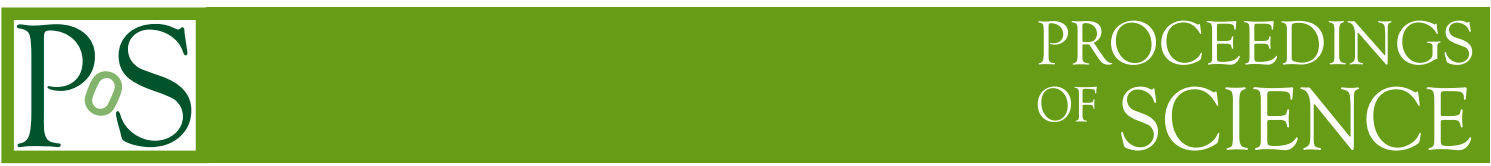

\title{
Radiative and electroweak penguin decay at Belle II
}

\author{
Keisuke Yoshihara, on behalf of the Belle II Collaboration \\ Department of Physics and Astronomy, Iowa State University, Ames, IA 50011, USA \\ E-mail: Keisuke.Yoshihara@cern.ch
}

Flavor changing neutral current $b$ to $s(d)$ decays, particularly radiative and electroweak penguin processes, will continue to be of great interest as a probe to search for physics beyond the Standard Model. Lately, there have been several anomalies observed in the $b$ to $s$ transition at the LHCb experiment in the angular observable, $P_{5}$ ' and lepton-universality measurements. The Belle II experiment will take an important role in providing independent measurements for these anomalies. This article describes how the Belle II physics program can be complementary to that of LHCb, and the Belle II ongoing studies and prospects on radiative and electroweak penguin processes in early data and the final dataset of $50 \mathrm{ab}^{-1}$.

BEAUTY2020

21-24 September 2020

Kashiwa, Japan (online) 


\section{Introduction}

The Standard Model of particle physics (SM) is a well established theoretical framework. However, there still remain unanswered questions such as baryon asymmetry in universe or the origin of dark matter, naively indicating the necessity of physics beyond the SM (BSM). As $B$-meson decays via flavor changing neutral current (FCNC) are forbidden at tree-level in the SM, these decays only proceed through higher-order loop diagrams. Radiative and electroweak penguin processes shown in Figure 1 are typical example of such $B$-meson FCNC decays.

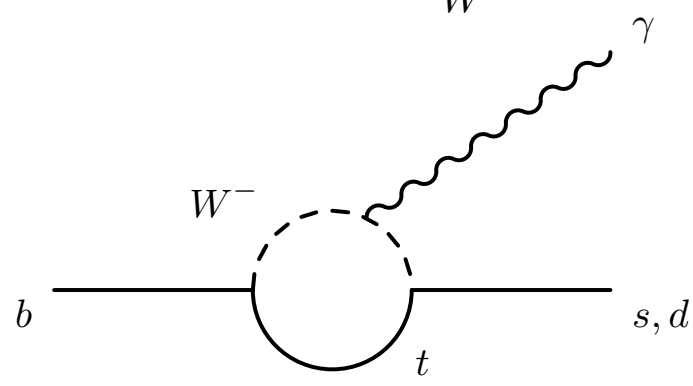

(a) radiative penguin

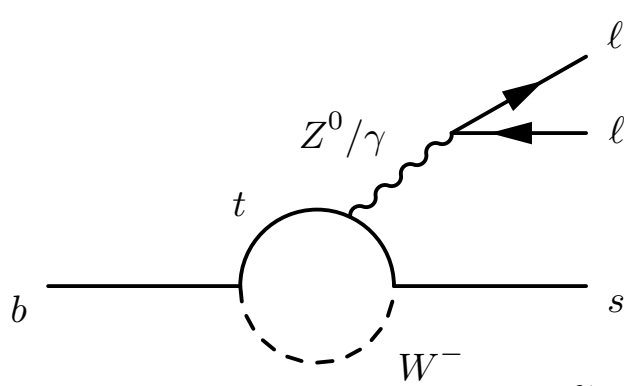

(b) electroweak penguin

Figure 1: The leading order Feynman diagrams for FCNC decays: (a) radiative penguin and (b) electroweak penguin process in the SM.

These penguin decays may receive contributions from BSM at a comparable size. Moreover, as the BSM appears as off-shell particles, these decays can provide a unique opportunity to explore higher mass scales compared to searches involving direct production. Several recent measurements in $b \rightarrow s \ell^{+} \ell^{-}$decays are in tension with the SM predictions. For example, a measurement of the decay-angular distributions in the decay $B^{0} \rightarrow K^{* 0} \mu^{+} \mu^{-}$performed by LHCb, shows more than $2.5 \sigma$ discrepancy with the SM for the observable $P_{5^{\prime}}$ [1,2]. Measurements of the ratio of branching fractions of the decays $B \rightarrow K^{(*)} \mu^{+} \mu^{-}$and $B^{+} \rightarrow K^{(*)} e^{+} e^{-}$, referred to as $R_{K}$ and $R_{K}^{*}$, are also found to be about $2.5 \sigma$ inconsistent with the lepton flavor universality [3,4]. The Belle II experiment will be able to provide an independent cross-check for these measurements, possibly finding a hint for BSM physics. Prospects for various measurements in the context of radiative and electroweak penguin decays are described in the following sections.

\section{SuperKEKB and Belle II detector}

SuperKEKB [5] is an $e^{+} e^{-}$collider, located at KEK in Tsukuba, Japan, with asymmetric beam energy of $4 \mathrm{GeV}$ for positrons and $7 \mathrm{GeV}$ for electrons such that the collision energy is equal to the energy of the $\Upsilon(4 S)$ resonance and the $B \bar{B}$ pair is boosted in the forward direction. SuperKEKB was designed and constructed as an upgrade of the KEKB accelerator complex, aiming to collect a total integrated luminosity of $50 \mathrm{ab}^{-1}$ with the maximum peak luminosity of $6 \times 10^{35} \mathrm{~cm}^{-2} \mathrm{~s}^{-1}$, which is approximately 30 times higher than the recorded peak luminosity of KEKB. This dataset will contain approximately $50 \times 10^{9} B \bar{B}$ events. The beam interaction point is surrounded by 
the Belle II detector [6], a multipurpose detector with a $4 \pi$ solid angle composed of various subdetector systems: pixel and silicon strip layers (PXD and SVD), central drift chamber (CDC), particle identification counters (TOP and ARICH), electromagnetic calorimeter (ECL), and $K_{L^{-}}$ muon detector (KLM). The Belle II detector was also designed and constructed as an upgrade of the Belle detector to be capable of reconstructing vertices of $B$-mesons precisely (vertex resolution of $<50 \mu \mathrm{m}$ is required) while coping with the higher luminosity as well as the higher beam-induced background. Belle II detector started taking physics data in 2019 and the total integrated luminosity of $74 \mathrm{fb}^{-1}$ was collected to date (September 2020).

\section{Analysis strategy}

\subsection{Theoretical framework}

The effective Hamiltonian is a general expression to describe a full theory at low energy $(\mu)$ and it has been used widely to interpret results in a model independent way. The Hamiltonian can be factorized into local operators $O_{i}$ with Wilson coefficients $C_{i}$ as follows:

$$
\mathcal{H}_{\mathrm{eff}} \sim \Sigma_{i} \mathcal{C}_{i}(\mu) O_{i}(\mu)
$$

There are 24 operators in the full expression. However, only three operators are relevant in the $b \rightarrow s$ process: the radiative penguin process involves $O_{7}$ while the electroweak penguin process involves $O_{9}$ (vector) and $O_{10}$ (axial-vector). The BSM contribution is expected to modify the SM contributions or introduce new operators in the form of $\Delta \mathcal{H} \sim\left(C_{B S M} / \Lambda_{B S M}\right) O_{B S M}$ where $\Lambda_{B S M}$ characterizes the energy scale of new physics. A global fit with all available measurements is able to obtain model-independent constraints on $C_{i}$ and hence constrain BSM models.

\section{2 $B$-meson reconstruction}

As $B$-mesons are pair-produced, Belle II will be able to perform inclusive measurements where the hadronic part of the decay is not specified. The inclusive measurements are complementary to the exclusive ones as they tend to be less affected by uncertainties due to hadronic form-factors. For the reconstruction of $B \rightarrow X_{s} \gamma$ decays, for example, there are two methods used and they are often referred to as (1) sum-of-exclusive method and (2) fully-inclusive method. In the sum-of-exclusive method, the hadronic system is reconstructed from exclusive decays containing a kaon and pion(s). Hadronic candidates are then combined with a hard photon to reconstruct $B$-meson candidates. The $B$-meson is referred to as $B_{\text {sig. }}$. In the fully-inclusive method, the other $B$-meson referred to as $B_{\text {tag }}$ is also reconstructed to improve the $\mathrm{S} / \mathrm{B}$ ratio. $B_{\text {tag }}$ is classified further into three types of tag; (1) a fully reconstructed hadronic final state (hadronic tagging), (2) a fully reconstructed semi-leptonic decay (semi-leptonic tagging), and (3) only an energetic lepton (leptonic tagging) from the $B$-meson decay. These reconstruction methods have their own pros and cons, providing access to different observables. A full event interpretation (FEI) [7] is a new reconstruction algorithm developed for Belle II employing machine learning techniques; the reconstruction efficiency of $B_{\text {tag }}$ is expected to improve approximately by a factor of two in comparison to the efficiency obtained by the Belle algorithm. 


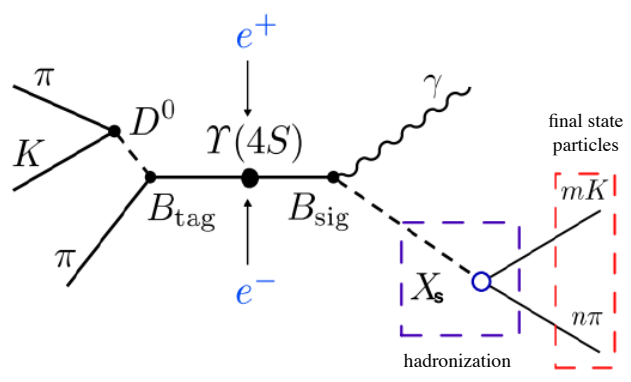

Figure 2: A schematic of a hadronically tagged decay of the $B$-meson $\left(B_{\text {tag }}\right)$ where $B \rightarrow D^{0}(\rightarrow K \pi) \pi$ is reconstructed in this example. Several hadronic decay chains with high branching fractions or efficiencies are included as $B_{\text {tag }}$. For the signal $\left(B_{\text {sig }}\right)$, the $B$-meson decay being targeted is reconstructed. The signal can include neutrinos as missing energy or an inclusive decay $\left(X_{S}\right)$ where all possible hadrons are considered.

\section{Radiative penguin decay}

$B \rightarrow X_{s} \gamma$ offers important constraints through $C_{7}$ on many BSM scenarios such as supersymmetry and models with extended Higgs sectors. The prompt photons are selected as isolated clusters in ECL that are not matched to any charged tracks. In order to reduce contaminations from asymmetric $\pi^{0} / \eta \rightarrow \gamma \gamma$ decays, each photon candidate is paired with all other photons in the event. The $B-$ mesons are then reconstructed as described in Section 3.2. The sensitivity projection for branching fraction, $B F\left(B \rightarrow X_{s} \gamma\right)$, is shown in Figure 3 .
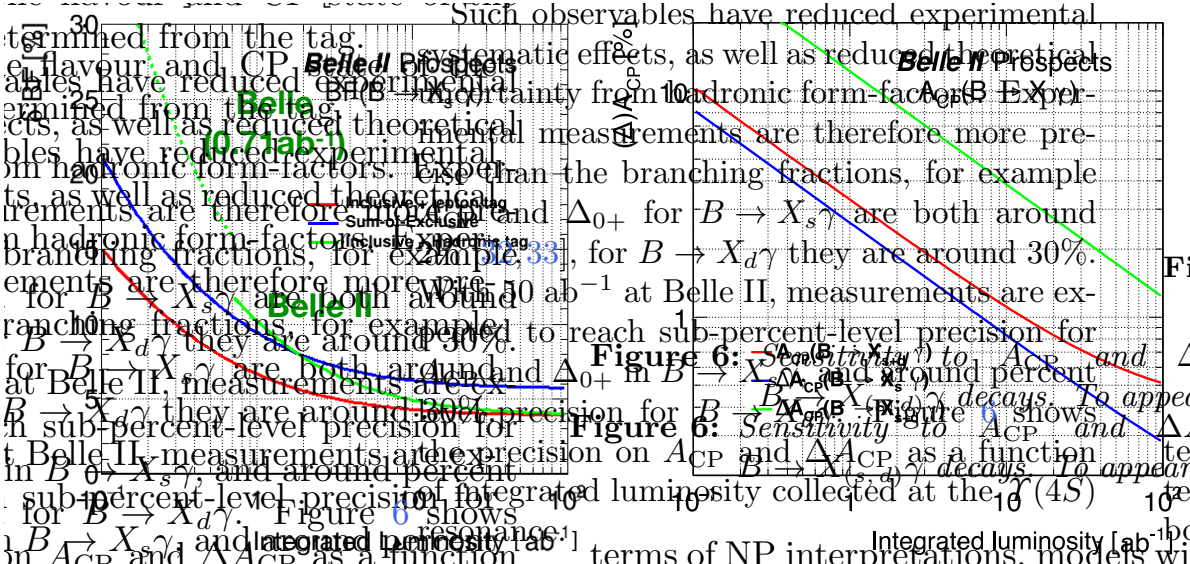

Figure 3: The Belle II sensitivity projection for $B F\left(B \rightarrow X_{s} \gamma\right)$ (left) and $(\Delta) \mathcal{A}_{C P}(B \rightarrow X \gamma)$ (right) as a function of integrated luminosity [8]. In the BF projection, the leptonic tag in the inclusive method (red), the sum-of-exclusive method (blue), and the hadronic tag in the inclusive method (green) are shown separately. The green line is discontinuous assuming the improvement of hadronic tagging in the Belle II experiment.

$B F\left(B \rightarrow X_{s} \gamma\right)$ will be measured to $6 \%$ precision with the early dataset of $5 \mathrm{ab}^{-1}$ and to $3 \%$ precision with the full dataset of $50 \mathrm{ab}^{-1}$. For the leptonic tagging, at present, the uncertainty is dominated by the systematic uncertainty arising from neutral hadrons faking photons. The uncertainty is expected to be reduced significantly at Belle II by exploiting the cluster shape information in ECL. For the hadronic tagging, the uncertainty was statistically dominated due to the low reconstruction 
efficiency of $B$-mesons. With the full dataset, the systematic uncertainty will be dominated by the mis-reconstruction of neutral hadrons as photons in the inclusive measurements. For the sum-ofexclusive method, the dominant uncertainty arises from hadronization of $X_{S}$ such as fragmentation and missing decay modes, and this uncertainty will remain dominant even with the full dataset. In addition to the $B F$ measurements, other important observables such as $\mathrm{CP}$ asymmetry $\left(\mathcal{A}_{C P}\right)$, isospin asymmetry $\left(\Delta_{0+}\right)$, and difference of $\mathrm{CP}$ asymmetry $\left(\Delta \mathcal{A}_{C P}\right)$ as defined in Equation 2 and 3 can be measured at Belle II.

$$
\begin{gathered}
\mathcal{A}_{C P}=\frac{\Gamma\left[\bar{B} \rightarrow X_{s} \gamma\right]-\Gamma\left[B \rightarrow X_{\bar{s}} \gamma\right]}{\Gamma\left[\bar{B} \rightarrow X_{s} \gamma\right]+\Gamma\left[B \rightarrow X_{\bar{s}} \gamma\right]}, \Delta_{0+}=\frac{\Gamma\left[B^{0} \rightarrow X_{s} \gamma\right]-\Gamma\left[B^{ \pm} \rightarrow X_{s} \gamma\right]}{\Gamma\left[B^{0} \rightarrow X_{s} \gamma\right]+\Gamma\left[B^{ \pm} \rightarrow X_{s} \gamma\right]}, \\
\Delta \mathcal{A}_{C P}=\mathcal{A}_{C P}\left[B^{ \pm} \rightarrow X_{s}^{ \pm} \gamma\right]-\mathcal{A}_{C P}\left[B^{0} \rightarrow X_{s}^{0} \gamma\right],
\end{gathered}
$$

In these measurements, experimental systematic uncertainties can be reduced along with theoretical uncertainty on hadronic form-factors. Figure 3 also shows sensitivity projection for these measurements.

\section{Electroweak penguin decay}

$B \rightarrow X_{s} \ell^{+} \ell^{-}$, where $\ell$ is either $e$ or $\mu$, also provides important information on the quark flavor sector that is complementary to the $B \rightarrow X_{s} \gamma$ decay, and constrains $C_{9}$ and $C_{10}$. Electrons are selected as an isolated track that is matched to a cluster in ECL with a large $p / E$. Charged tracks that leave cluster hits in the KLM are selected as muons. Belle II is capable of performing inclusive and exclusive measurements of the $B \rightarrow X_{s} \ell^{+} \ell^{-}$decay.
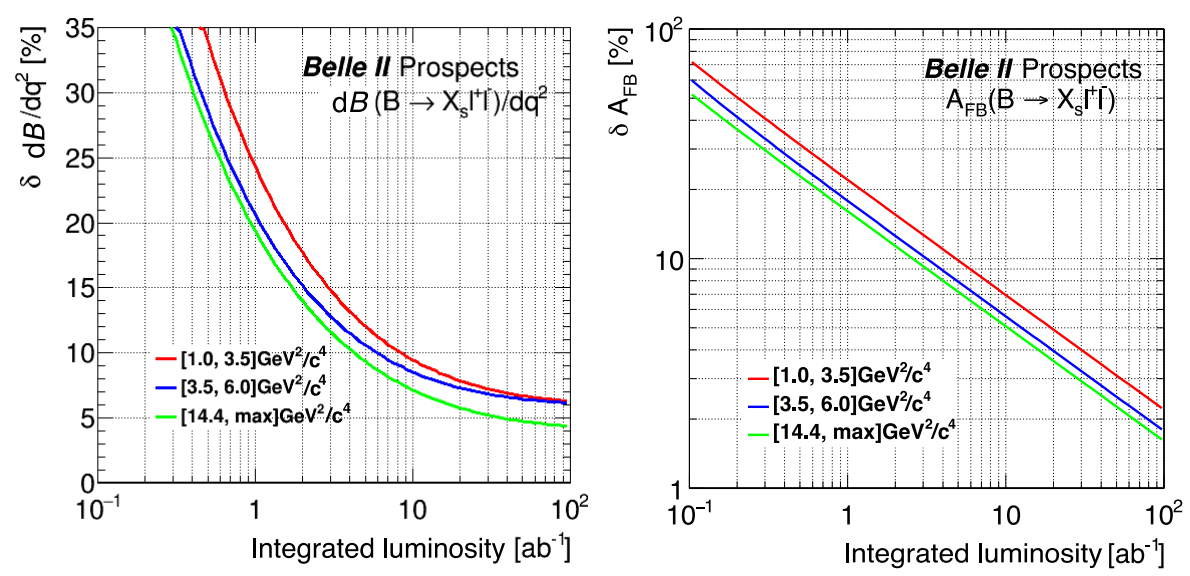

Figure 4: The Belle II sensitivity projection for $B F\left(B \rightarrow X_{s} \ell^{+} \ell^{-}\right)$(left) and $\mathcal{A}_{F B}$ (right) in three different regions of squared invariant mass of the lepton pair [8].

In the inclusive mode, hadronic uncertainties can be significantly suppressed compared to the exclusive mode, and hence higher experimental sensitivity is expected. The branching fraction of $B \rightarrow X_{s} \ell^{+} \ell^{-}$decay can be measured in three regions of squared invariant mass of the lepton 
pair. Figure 4 shows the sensitivity projection for the branching fraction measurement as a function of integrated luminosity. In addition to the $B F$ measurements, the forward-backward asymmetry $\left(\mathcal{A}_{F B}\right)$ and a difference of $\mathcal{A}_{F B}\left(\Delta_{C P}\left(\mathcal{A}_{F B}\right) \equiv \mathcal{A}_{F B}^{\bar{B}}-\mathcal{A}_{F B}^{B}\right)$ can be also measured as a function of the dilepton invariant mass at Belle II. The sensitivity projection for the $\mathcal{A}_{F B}$ measurement is also shown in Figure 4. The $\mathcal{A}_{F B}$ measurement is expected to provide the most stringent constraint on $C_{9}$ and $C_{10}$ as theoretical and experimental systematic uncertainties are largely canceled. The uncertainty on the $B F$ measurements will be dominated by theoretical uncertainties at $15 \mathrm{ab}^{-1}$ while the $\mathcal{A}_{F B}$ measurement will be statistically limited even at $50 \mathrm{ab}^{-1}$. Furthermore, $\mathcal{A}_{C P}$ can be measured at Belle II. The $\mathcal{A}_{C P}$ of the $B \rightarrow X_{s} \ell^{+} \ell^{-}$decay can be a sensitive probe for new sources of $C P$ violation. The test of lepton flavor universality is also an important observable. The $R(K)$ and $R\left(K^{*}\right)$ anomalies can be cross checked in the low- and high- $q^{2}$ regime with higher sensitivity than in previous measurements by taking an advantage of the high reconstruction efficiency and purity for both electrons and muons. In addition, Belle II will perform an inclusive $R_{X_{s}}$ measurement, which is expected to help largely cancel systematic uncertainties. Moreover, angular observables such as $P_{5}$ ' can be measured in low- and high- $q^{2}$ regime and another lepton universality test can be performed with $Q_{5}\left(\equiv P_{5}^{\prime e}-P_{5}^{\prime \mu}\right.$ ) observable. The expected sensitivity in $R_{X_{s}}$ and $P_{5^{\prime}}$ (and $Q_{5}$ ) as a function of the integrated luminosity is shown in Figure 5.
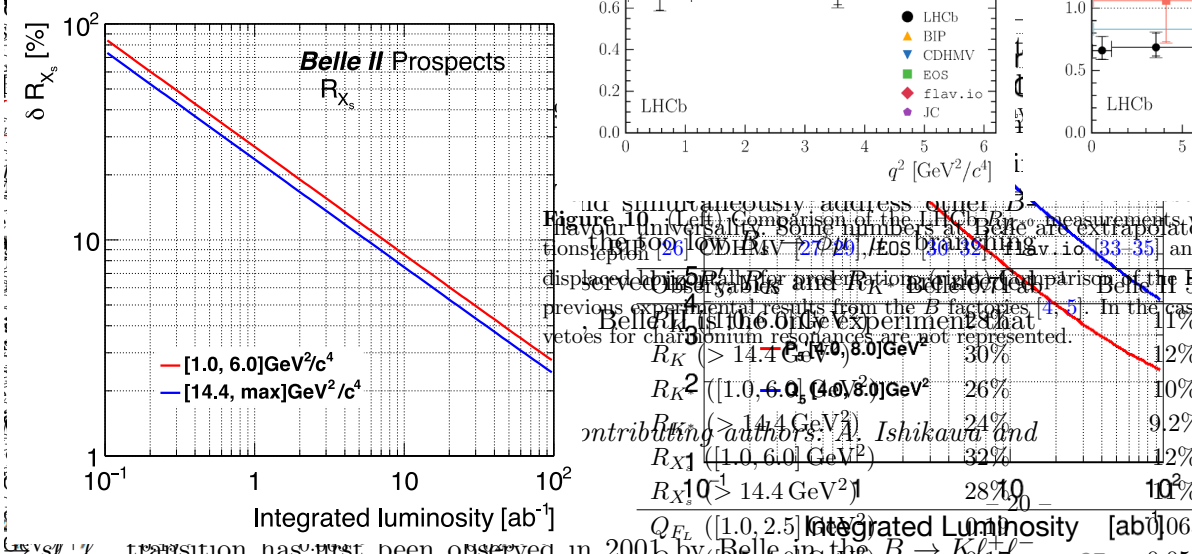

Figure 5: The Belle II sensitivity projection for $R_{X_{s}}$ (left) and $P_{5}^{\prime}$ (and $Q_{5}$ ) (right) as a function of integrated luminosity [8].

\section{6. $B \rightarrow K^{(*)} v \bar{v}$ decay}

$B \rightarrow K^{(*)} v \bar{v}$ is another probe that is sensitive to $C_{9}$ and $C_{10}$. It is theoretically and experimentally cleaner than $B \rightarrow X_{S} \ell^{+} \ell^{-}$because of the absence of a photon mediated contribution. The $B \rightarrow$ $K^{(*)} v \bar{v}$ decay has never been observed experimentally. Belle II is expected to be the first experiment to observe the decay mode with $5 \mathrm{ab}^{-1}$ assuming the SM rate. With the full dataset, the branching fraction can be measured to about $10 \%$ accuracy. Since two neutrinos are present in the final state, the other $B$-meson needs to be fully reconstructed. The FEI algorithm described above is expected to play an essential role in this measurement. The fraction of longitudinal polarization $\left(F_{L}\right)$ is also known to be sensitive to the BSM signature and the detailed study can follow with the full dataset. 


\section{Conclusion}

Rare radiative and electroweak processes have drawn a lot of attention from the high energy physics community because of several anomalies observed in previous measurements (mainly performed by $\mathrm{LHCb}$ ) in the $b \rightarrow s$ transition. The SuperKEKB / Belle II experiment started physics data-taking in 2019 and $74 \mathrm{fb}^{-1}$ has been collected to date (September 2020). Belle II will have access to decay modes and measurements that are likely challenging at $\mathrm{LHCb}$, such as the $B \rightarrow K^{(*)} v \bar{v}$ decay and various inclusive measurements, and will be able to provide an independent determination of these anomalies, possibly finding a hint for BSM in the near future.

\section{References}

[1] LHCb Collaboration, "Angular analysis of the $B^{0} \rightarrow K^{* 0} \mu^{+} \mu^{-}$decay using $3 \mathrm{fb}^{-1}$ of integrated luminosity", JHEP02 (2016)104.

[2] LHCb Collaboration, "Measurement of CP-Averaged Observables in the $B \rightarrow K^{0} \mu^{+} \mu^{-}$Decay", PRL125.011802(2020).

[3] LHCb Collaboration, "Test of lepton universality with $B^{0} \rightarrow K^{* 0} \ell^{+} \ell^{-}$decays", JHEP08(2017)055.

[4] LHCb Collaboration, "Search for lepton-universality violation in $B^{+} \rightarrow K^{+} \ell^{+} \ell^{-}$decay", PRL122.191801(2019).

[5] Y. Onishi et al, "Accelerator design at SuperKEKB", https://doi.org/10.1093/ptep/pts083

[6] Belle II Collaboration, "Belle II Technical Design Report", arXiv:1011.0352.

[7] T. Keck et al., "The Full Event Interpretation - An exclusive tagging algorithm for the Belle II experiment", Comput. Softw. Big Sci. 3 (2019), arXiv:1807.08680 [hep-ex].

[8] Belle II Collaboration, "Belle II Physics Book", PTEP2020.029201(2020), https://arxiv.org/abs/1808.10567. 